\title{
Comparison Analysis of Gait Classification for Human Motion Identification Using Embedded Computer
}

\author{
Agung Nugroho Jati, Astri Novianty, Nanda Septiana, Leni Widia Nasution \\ Department of Computer Engineering, School of Electrical Engineering, Telkom University, Indonesia
}

\begin{tabular}{l}
\hline \hline Article Info \\
\hline Article history: \\
Received Jan 26, 2018 \\
Revised Jun 28, 2018 \\
Accepted Jul 22, 2018 \\
\hline Keyword: \\
Gait Recognition \\
Human Motion Identification \\
K-Nearest Neighbour \\
Support Vector Machine
\end{tabular}

Corresponding Author:

Agung Nugroho Jati,

Department of Computer Engineering,

School of Electrical Engineering, Telkom University,

Jl. Telekomunikasi Terusan Buah Batu, Bandung, 40257, Indonesia.

Email: agungnj@telkomuniversity.ac.id
Copyright (C) 2018Institute of Advanced Engineering and Science. All rights reserved.

\section{INTRODUCTION}

Every single person in the world has unique features which is different from others. In engineering, it can be used as a key for identification or in security system. Generally, it's called as biometric system. Many implementation of biometric system use part of human body, but it's still very rare biometric system use human motion. Most of them, mainly used face recognition such as to define gender of human identification [1]. So in this paper, it will be disscused about methods for identifying human feature based on human motion especially using human gait.

Gait is defined as the way an individual organism walk, including human. And every human has a unique gait as a behavioral characteristic which is different from each other. It depends on weight, leg's length and size, and posture of body [2]. Studying gait combines more than a subject, like medical studies, psychology, biology, and motion analysis [3]. It strongly proves that gait can be used in biometrics system.

To recognize and classify the human gait, there are two kind of approach. First one, it's based on motion analysis which needs attention of whole human body movement. To ease these methods, we can use captured image of human silhouttes. Second, it's based on feature approach which only needs to pay attention in the spesific part of human body, especially the moving part like knees and hinges [4], [5]. In other research, it's said that there two approach of gait analysis, spatial feature and temporal feature analysis [6].

Comparing the original human body model with simulation model of gait feature can be used to know someone's gait characteristic. Simulation model contains a gait cycle which is a periodic condition from a foot stepping in a ground until another turns. The cycle is divided into two parts of condition, which are called stance and swing. A stance is described when only a foot stepping in a ground, and swing defines when a foot swinging in the air. Generally, a cycle consists of $60 \%$ stance and $40 \%$ swing [7]. 
It needs some steps based on image processing for identifying human gait. First, motion must be estimated in a lot of number in an area and must be limited by human anatomic constraint in hierarchical model. It's needed to extract the fixed human body parameters. In the next step, the form of human gait will be analyzed by using edge sharpness to representate legs area. Heuristic model is used to estimate the period and will be compared with unique form of gait cycle as shown in Figure 1 [8]. And the last, that unique form will be recognized by classification methods. To ease, we can use HOG and PCA for extracting gait feature.

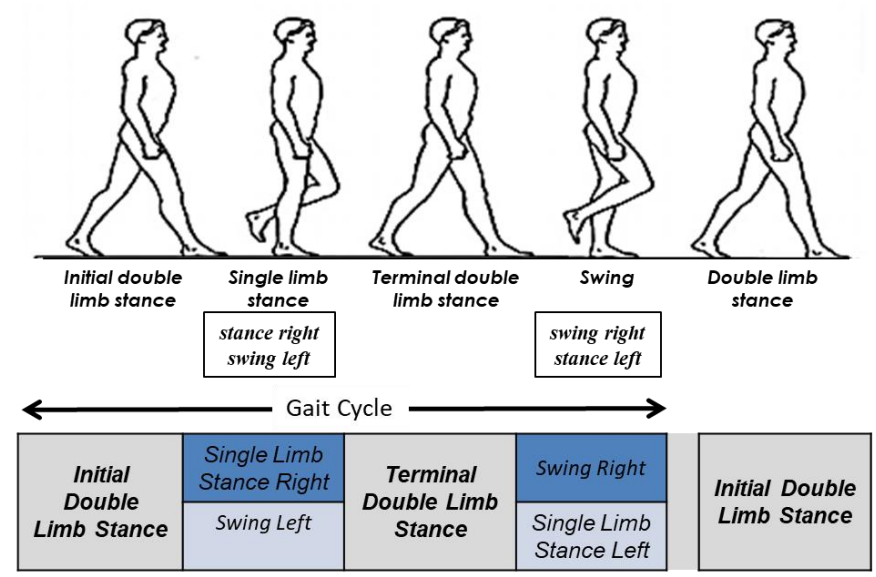

Figure 1. Periodic gait cycle [8]

Histogram of Gradient (HOG) is an algorithm which is used to detect the spesific object in an static image. HOG utilizes colour changing of each pixel with others in the digital image and well-known as gradient [9]. While Principal Component Analysis (PCA) is used to identify the pattern and express them to the other form in order to show the differences and the similarities. PCA is often used as feature extraction method [10]. Indeed, PCA has ever been used to extract the features from chest X-ray image and provided more than $95 \%$ accuracy [11].

In this paper, will be focused only in classification methods. The classification methods have been compared in this paper are K-nearest Neighbour (KNN) and Support Vector Machine (SVM). This comparison is needed for our future work in implementation of Human Identification System based on embedded computer. The system accuracy in the main problem of gait recognition. Besides, it's needed to know how much resources used for computation. SVM is chosen because the concept of multiclass one againts one can decrease the classification error [2]. On the other hand, KNN is a simple method in classification which uses the concept of learning by analogy and only classifies based on most matched features [12].

This paper will be divided into four sections which in the first, discussed about the review of human gait, the main focus of the discussion, and some of related works. In the second section, shown of the techniques used in the research. The result will be provided in the section four. And in the last, will be presented the conclusion and the future work of the research.

\section{RESEARCH METHOD}

In general, gait identification process can be described as follows. General Process Design as shown Figure 2. Image aqcuisition is the first step to get digital image. In this step, camera will capture video and divides into frames. Frames will be entered into next step, called preprocessing. In pre processing, images will be processed to increase image quality, noise reduction, transform the image into other format, and determine the part will be observed. The processes are start rom grayscaling, thresholding, background substraction, closing, and segmentation. 


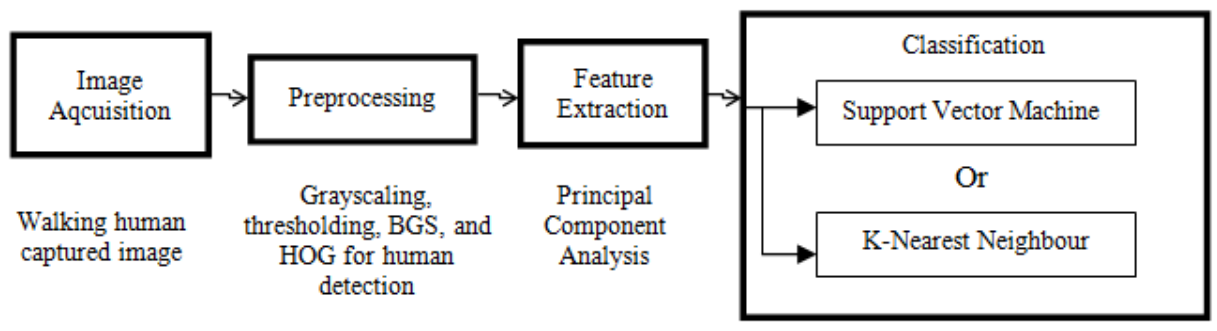

Figure 2. General Process Design

Feature extraction is proposed to get the important informations from the image to define the differences form others. To extract them, Principal Component Analysis (PCA) is used in this research. PCA, which first was introduced by Pearson (1901) and Hotelling (1933), is designed to collect the unique features from the image [13]. The unique features can be found by transforming the image from high dimensional data into low one. It's obtained from a set training data of the image [14]. To simplify the problem, each acquired image was rezised into 80x80 pixel image. PCA steps will be provided in the Table 1 below.

Table 1. PCA Processes for Feature Extraction

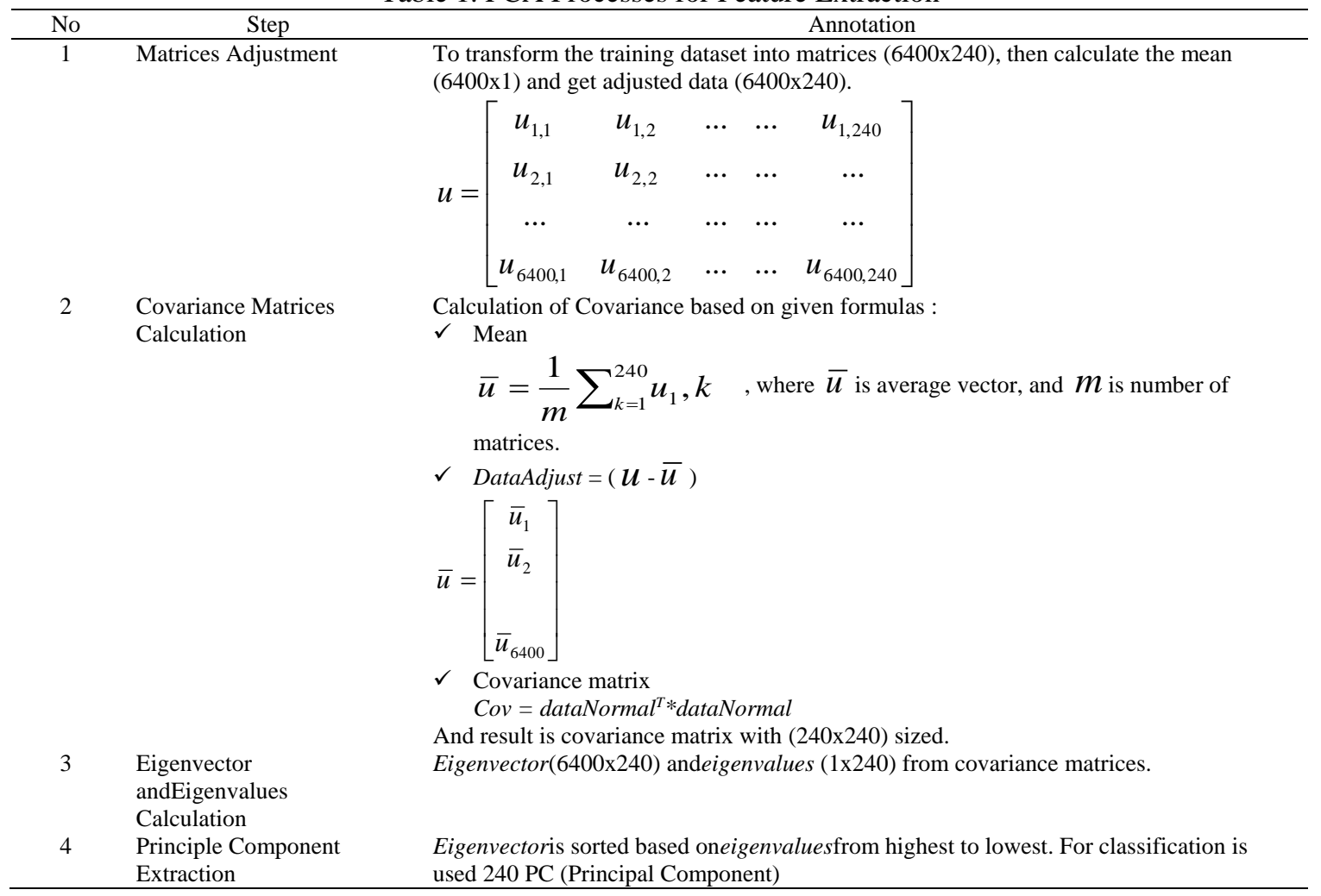

Result data from feature extraction was used to classify and recognize gait and its owner. As explained before, main focus in this research is the comparison between SVM and KNN for classification and identification method. However, they shared same data and tested by the same scenarios.

\subsection{Support Vector Machine (SVM) for Gait Classiffication}

SVM will define the divisor between two kind of classes in the input space known as hyperlane. Hyperlane can be find out by calculate its margin and find maximum value. There is support vector defined as closest data for each class. It can be used kernel approach to get them. Kernel is defined as function for mapping data feature from the origin dimension into other feature with highest dimension [15]. 
There are two kind of SVM functions in classification. The first is needed to define the classes by using training process. It's purpose is to find w (weight), $\mathrm{b}$ (bias), and support machine from each class. Then in the testing process, parameters which have been obtained from training process include in the calculation as input to make decision function.

$$
f(x)=y_{i} a_{i} x_{i} x+b^{*}
$$

Sign value from processes above is the result from tested data [16]. Refering to the training process, its implementation uses Gaussian kernel and Sygma value 6000. In the previous research, it shows the maximum accuracy with optimum computation time. As we know that kernel type used is very important for all of system performance.

\subsection{K-Nearest Neighbour (KNN) for Gait Classification}

K-Nearest Neighbour (KNN) is well-known supervised learning algorithm, which classifies objects based on the majority class of features from $\mathrm{k}$ amount of closest neighbour. KNN is based on learning by analogy concept, where learning data are described by $\mathrm{n}$ dimensional numerical attributes. Each of them represents a point in $\mathrm{n}$ dimensional space. Euclidean distance formula is used to calculate the distance between query data and learning data [12], [17], [18].

$$
D_{\text {euc }}(P, Q)=\sqrt{\sum_{i=1}^{n}\left(p_{i}-q_{i}\right)^{2}}
$$

KNN classification accuracy depends on similarity measurement usage and value of $\mathrm{k}$. Before implementation, have been tested some varinace of $\mathrm{k}$ in order to get the best accuracy. They are 1, 3, 5, 7 . And for implementation, used $\mathrm{k}=1$ because this value has best accuracy.

\section{RESULTS AND ANALYSIS}

In this section, it is explained the results of testing scenarios. The parameters which is measured and analyzed are system accuration, computation time, light depedency for accuration, and distance of camera.

\subsection{System Accuracy}

Based on previous section, there are two type of classification methods. They are Support Vector Machine (SVM) and K-Nearest Neighbour (KNN). In SVM, it has been chosen some variables value, sigma 6000 with Gaussian kernel. Then, in KNN method there's given k=1 for classification. It's based on the non real time testing for each method in order to take the best performance for each classification method. Comparison of KNN and SVM Accuration as shown in Figure 3.

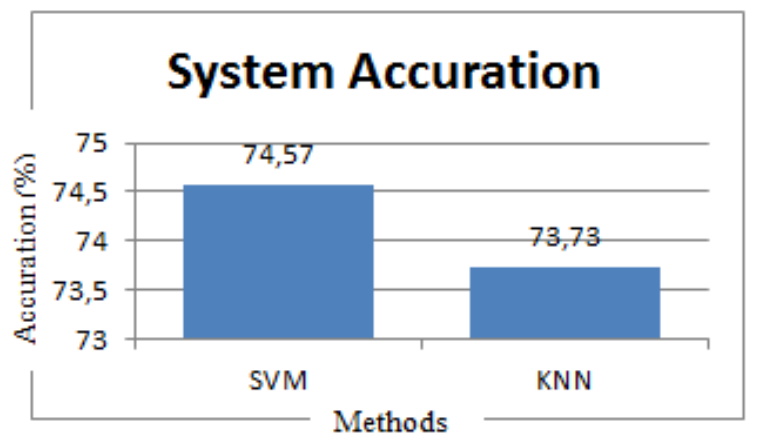

Figure 3. Comparison of KNN and SVM Accuration

In the figure above, shown that classification using SVM was more accurate than KNN. The testing was done by using 118 data, and used 6 classes. SVM became more accurate because it classified by finding best hyperlane to divide data and based on "multiclass one againts one" concept. While KNN only used k=1 which means it only compares with less amount of neighbourhood data. 


\subsection{Computation Time}

Computation time analysis was performed in order to know the average of processing time. Besides, it's needed to compare between SVM and KNN what's faster in process. This measurement used same condition with previous test which was used sigma 6000 and Gaussian kernel for SVM, and k=1 for KNN. The result is shown in Figure 4 below.

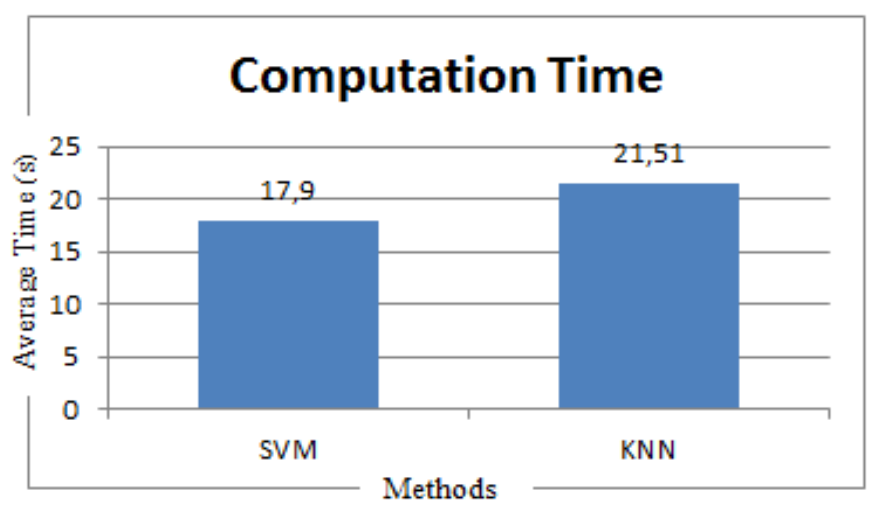

Figure 4. Comparison of KNN and SVM Computation Time

SVM gives faster result than KNN because in SVM only processes vector, while KNN compares some neighbour data to find similarities. SVM needs average 17,9 s to compute them, and KNN needs average $22,51 \mathrm{~s}$.

\subsection{Light Dependency}

In order to analyze the light effect in processing, this test was proposed. The test uses six classes come from six person gait. Each class uses fourthy trainning data and eight test data. The result only represent the real time testing, means come from eight test data. Classification method parameters still use same with other test. For SVM still use Gaussian Kernel with 6000 sigma, while KNN uses k=1. Accuration in Day Light Measurement as shown in Figure 5. Accuration in Night Light Measurement as shown in Figure 6.

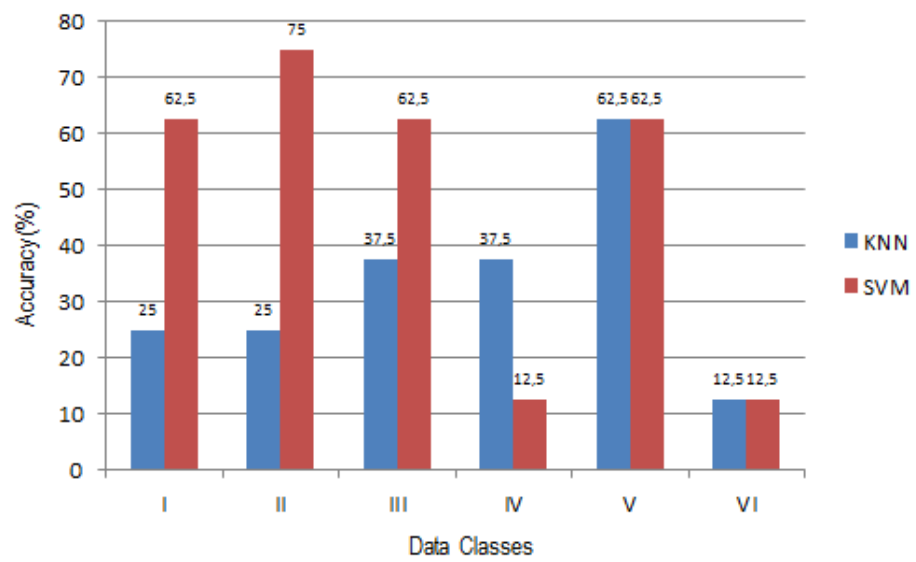

Figure 5. Accuration in Day Light Measurement 


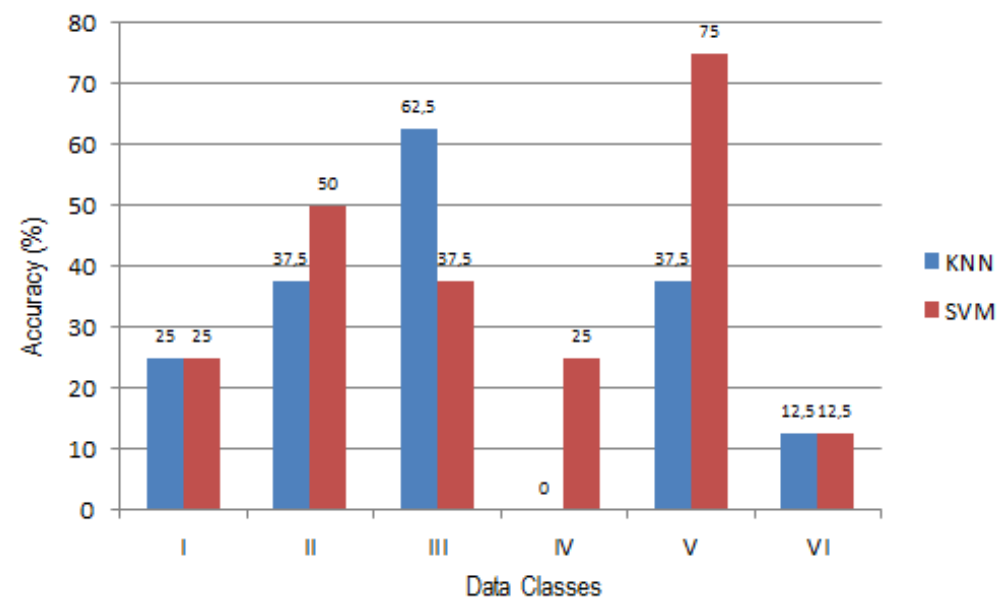

Figure 6. Accuration in Night Light Measurement

Based on the test, it's shown that generally higher luminance will decrease the accuracy. The higher light intensity will make the data have more noise than normal condition. Besides, lamp will produce shadows. It makes in the night, accuracy is decrease.

\subsection{Effect of Camera Positions}

In this test, known that position and distace of camera from the object influenced the accuracy. It's caused by pattern of captured frames. Closer object from the camera causes captured image contain less background but not full object in the frame. While further distance makes the object in the frame sized smaller. So, it needs optimum distance. Result can be described in the graphic below.

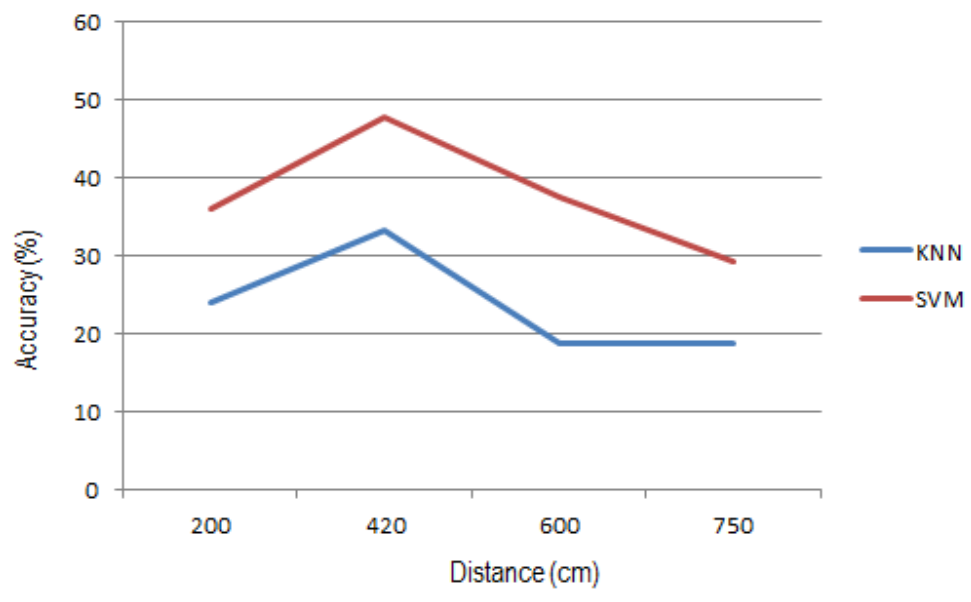

Figure 5. Accuration in Day Light Measurement

\section{CONCLUSION}

Based on the implementation, testing, and analysis can constructed some conclusions of the study. Overall, SVM is better than KNN for classifying human motion by using gait of them. It can be shown in accuracy, computation time, and some effect of changed condition parameters. Altough, human identification by using gait has too many constraints to be considered. Actually in implementation, it needs more parameters to make better accuracy.

In the further stage of study, the result from this study can be considered to be implemented in single board computer, such as raspberrry pi, pcduino board, beaglebone, etc as a security monitoring system. It can be integrated to our movement prediction system which has better result in accuracy [19]. Although, it needs more measurement, especially in resource allocation and management. 


\section{REFERENCES}

[1] M.J. Barani, K. Faez, F. Jalili. "Implementation of Gabor Filters Combined with Binary Features for Gender Recognition". International Journal of Electrical and Computer Engineering (IJECE). Vol 4 (1) : 108-115. February 2014.

[2] Darma Putra. Biometrics System. Yogyakarta: Andi Publisher. 2009.

[3] Anil Jain, Ruud Bolle, Sharath Pankati. "Biometrics Personal Identification in Networked Society". Kluwer Academic Publishers.

[4] David Post. Gait Analysis Review. Downloaded from www.nd.edu/ dpost/IntSyst/report1.pdf. 2006.

[5] Chiras Ben Abdelkader, Ross G. Cutler, dan Larry S. Davis. "Gait Recognition using Image Self Similiarity". Hindawi Publishing Corporation. 2004.

[6] Zhang Zhaoxiang, Maodi Hu, Yunhong Wang. “A survey of Advances in Biometric Gait Recognition”. Journal. China: Beihang University.

[7] Spencer, N.M. "Pose Invariant Gait Analysis and Reconstruction". Faculty of Engineering, Science and Mathematics, School of Electronics and Computer Science, University of Southamton. 2005.

[8] Nash, J.M., Carter, J.N., and M.S., Nixon. "Dynamic feature extraction via the velocity trhough transform". Pattern Recognition Letters. vol 18 pp 1035- 1047. 1997.

[9] Fathurrahman, Irwan. "Implementation of Text Detection and Recognition by Using Histogram of Oriented in Android Platform". Final Project. Bandung: School of Electrical and Informatics, Institut Teknologi Bandung. 2012. (unpublished)

[10] Dian Esti Pratiwi. "Implementation of Face Recognition by using (Principal Component Analysis)". Final Project. Yogyakarta: Electrical and Instrumentation Program, Universitas Gadjah Mada. 2013. (unpublished)

[11] Roopa H, Asha T. "Feature Extraction of Chest X-ray Images and Analysis Using PCA and kPCA". International Journal of Electrical and Computer Engineering (IJECE). Vol 8 No 5. October 2018.

[12] Wahyu Hidayat. "K-Nearest Neighbor Implementation for Landscape Image based on Colour Feature and Texture". Final Project. Bandung: Institut Teknologi Telkom. 2007. (unpublished)

[13] J.S. Butzer, A.P.H. Butler, P.J. Bones. "Medipix Imaging Evaluation of Datasets with PCA". Image and Vision Computing New Zealand IEEE International Conference Proceedings. 2008; 23: 1-6.

[14] J.A. Lee, M. Verleysen. "Unsupervised Dimensionality Reduction: Overview and Recent Advances". IEEE International Joint Conference on Neural Networks. 2010; 1-8.

[15] Ching-Chih Sai, You-Zhu Chen, Ching-Wen Liao. "Interactive Emotion Recognition using Support Vector Machine for Human-Robot Interaction”. IEEE International Conference on System, man and Cybernetics. 2009; 407-412.

[16] Yang-Guang Liu, Qi Chen, Rui-Zhao Yu. "Extract Candidates of Support Vector Training Set". IEEE International Conference on Machine Learning and Cybernetics. 2003; 5: 3199-3202.

[17] J.L. Henry. A. "K-Nearest Neighbour Method for Managing the Evolution of a Learning Base". IEEE International Conference on Computational Intelligence and Multimedia Applications. 2001; 4: 357-361.

[18] Zhongjian Song, Qing Wu, Chunming Xia. "Pattern recognition of Finger-Motions based on Diffusioan Maps and Fuzzy K-Nearest Neighbour Classifier”. IEEE International Conference on Signal Processing. 2012; 2: 1207-1212.

[19] AN Jati, L. Novamizanti, M.B. Prasetyo, A.R. Putra. "Evaluation of Moving Object Detection Methods based on General Purpose Single Board Computer". Indonesian Journal of Electrical Engineering and Computer Science (IJEECS). Vol 4 (1) : 123-129. April 2015. 